\title{
Algoritmo LMS de Passo Variável para Ambientes com Razão Sinal-Ruído Baixa
}

\author{
José Gil F. Zipf, Orlando J. Tobias e Rui Seara
}

\begin{abstract}
Resumo-Este trabalho apresenta um novo algoritmo LMS de passo variável para ambientes com razão sinal-ruído (SNR) baixa. Em algoritmos de passo variável, tanto o método de ajuste de passo quanto o desajuste do algoritmo são afetados pelo ruído de medição. Dessa forma, o uso da medida de autocorrelação do sinal de erro $\operatorname{lag}(1)$ vem provando ser eficaz para o ajuste do passo, apresentando uma aceitável imunidade para níveis de ruído não muito altos, contudo, falhando para valores baixos de SNR. O método proposto utiliza um procedimento similar ao anterior, porém agora considerando diversos valores de atrasos para obter a autocorrelação do sinal de erro. Tal estratégia resulta em uma substancial melhora no desempenho do algoritmo para níveis de ruído de medição altos à custa de um pequeno acréscimo na carga computacional. Simulações numéricas confirmam o bom desempenho do algoritmo proposto.
\end{abstract}

Palavras-chave-Algoritmos adaptativos em ambientes com razão sinal-ruído baixa, algoritmo LMS de passo variável, medida de autocorrelação do sinal de erro.

Abstract-This paper presents a new variable step-size (VSS) LMS algorithm for low signal-to-noise-ratio (SNR) environment. In VSS algorithms, both the step-size adjustment method and the algorithm misadjustment are affected by the measurement noise. Thereby, the use of the lag(1) error signal autocorrelation measurement has proved to be effective for step-size adjustment, providing an acceptable noise immunity for medium noise levels; however, failing for small values of SNR. The proposed approach uses a procedure similar to the previous one, but now considering several values of lags for obtaining the error signal autocorrelation. Such a strategy results in a significant improvement in the algorithm performance for high measurement noise levels at the expense of a small increase in the computational cost. Numerical simulations confirm the good performance of the proposed algorithm.

Keywords-Adaptive algorithms in low signal-noise-ratio environment, variable step-size LMS algorithm, error signal autocorrelation measurement.

\section{INTRODUÇÃO}

O algoritmo LMS é o mais popular algoritmo utilizado em aplicações de filtragem adaptativa devido à sua baixa complexidade computacional e sua muito boa característica de estabilidade [1]. Tais vantagens tornam o algoritmo LMS

José Gil F. Zipf, Orlando J. Tobias e Rui Seara, LINSE - Laboratório de Circuitos e Processamento de Sinais, Depto. de Eng. Elétrica, Universidade Federal de Santa Catarina, Florianópolis, SC, E-mails: \{gil, orlando, seara\}@linse.ufsc.br.

José Gil F. Zipf e Orlando J. Tobias são também Professores do Departamento de Engenharia Elétrica e Telecomunicações da Universidade Regional de Blumenau (FURB), Blumenau, SC, Brasil.

Este trabalho foi parcialmente financiado pelo Conselho Nacional de Desenvolvimento Científico e Tecnológico (CNPq). adequado para aplicações de identificação de sistemas, cancelamento de ruído, cancelamento de eco, equalização de canal, dentre outras [2]. O algoritmo LMS convencional considera um passo de adaptação fixo. A escolha desse parâmetro afeta sobremaneira o desempenho do algoritmo. Assim, um valor elevado do passo leva a uma velocidade de convergência maior associada também a um maior desajuste do algoritmo. Por outro lado, um valor pequeno do passo de adaptação resulta em um menor desajuste do algoritmo às custas de uma taxa de convergência mais baixa. Para superar tal compromisso, são propostos na literatura os algoritmos de passo variável, os quais visam obter uma alta velocidade de convergência aliada a um reduzido desajuste do algoritmo. Para o caso do algoritmo LMS, tais abordagens são denominadas algoritmos VSSLMS (variable step-size LMS). A idéia central nesse tipo de algoritmo é utilizar um valor grande de passo de adaptação no início do processo de convergência e ir gradualmente (segundo algum critério dado) reduzindo o valor do passo à medida que o regime permanente se aproxime. As estratégias de ajuste do passo de adaptação nos algoritmos VSSLMS são baseadas em diferentes princípios: gradiente do erro quadrático [3]-[9], erro quadrático instantâneo [10]-[12], função de autocorrelação do erro [13], erro de adaptação absoluto [14], normalização do vetor de erro [15], valor absoluto do vetor de coeficientes [16]-[18], e outros métodos não nomeados aqui [19]. Em [10], é introduzido um algoritmo VSSLMS baseado no erro quadrático instantâneo. Esse algoritmo, denominado algoritmo de Kwong, apresenta um bom desempenho para um grande número de aplicações práticas. No entanto, o processo de ajuste do passo de adaptação como também o desajuste do algoritmo são afetados fortemente pelo ruído de medição. Em [13], é proposta uma modificação ao algoritmo de Kwong. Esse algoritmo (chamado algoritmo de Aboulnasr) apresenta uma considerável melhora de imunidade ao ruído de medição. Ele é baseado na medida de autocorrelação do sinal de erro $\operatorname{lag}(1)$. Neste trabalho, uma versão melhorada do algoritmo de Aboulnasr em termos de imunidade ao ruído de medição é proposta. Aqui, a idéia básica para ajustar o parâmetro de passo é considerar as diversas funções de autocorrelação do sinal de erro $\operatorname{lag}(1), \operatorname{lag}(2), \ldots, \operatorname{lag}(N)$, com $N$ denotando a ordem do filtro adaptativo. É importante ressaltar que o uso de vários lags aumenta apenas marginalmente a complexidade computacional do algoritmo. Através de simulações numéricas, é verificado o bom desempenho do algoritmo aqui proposto. 


\section{Algoritmos VSSLMS}

O uso de uma estratégia VSS melhora o desempenho do algoritmo LMS convencional. Assim, nesta seção, serão descritos brevemente os algoritmos de Kwong e de Aboulnasr, que são fortemente relacionados ao algoritmo proposto neste trabalho. Para tal descrição, é considerado um esquema de identificação de sistemas, o qual está apresentado na Fig. 1.

Nesta figura, o sinal de saída do sistema desconhecido, corrompido por um ruído de medição, é dado por

$$
d(n)=\mathbf{w}_{\mathrm{o}}^{\mathrm{T}} \mathbf{x}(n)+\eta(n)
$$

onde $\mathbf{x}(n)=[x(n) x(n-1) \cdots x(n-N+1)]^{\mathrm{T}}$ denota o vetor de entrada, sendo $\{x(n)\}$ um processo gaussiano de média zero e variância $\sigma_{x}^{2}, \eta(n)$ é um ruído de medição i.i.d com variância $\quad \sigma_{\eta}^{2}, \quad \mathbf{w}(n)=[w(n) w(n-1) \cdots w(n-N+1)]^{\mathrm{T}} \quad$ é $\quad$ o vetor de coeficientes e $\mathbf{w}_{\mathrm{o}}=\left[w_{0,1}(n) w_{0,2}(n-1) \cdots\right.$ $\left.w_{0, N-1}(n-N+1)\right]^{\mathrm{T}}$ denota a planta do sistema a ser identificado. $\mathrm{O}$ sinal de erro é dado por

$$
e(n)=d(n)-\mathbf{w}^{\mathrm{T}}(n) \mathbf{x}(n) .
$$

A expressão de atualização do vetor de coeficientes para os algoritmos VSSLMS é escrita como

$$
\mathbf{w}(n+1)=\mathbf{w}(n)+\mu(n) e(n) \mathbf{x}(n)
$$

onde $\mu(n)$ é o passo de adaptação variável. Para garantir uma operação estável do algoritmo adaptativo, os limites de ajuste do passo de adaptação são fixados pela seguinte inequação [2]:

$$
0<\mu(n)<\frac{2}{3 \operatorname{tr}[\mathbf{R}]}
$$

onde $\mathbf{R}$ é a matriz de autocorrelação do vetor de entrada.

\section{A. Algoritmo de Kwong}

Este algoritmo utiliza o erro quadrático instantâneo para atualizar o passo de adaptação, o qual é expresso como

$$
\mu(n+1)=\alpha \mu(n)+\gamma e^{2}(n)
$$

onde $\alpha>0$ e $\gamma>0$ são parâmetros de controle do algoritmo.

A principal característica deste algoritmo é que valores elevados de erro aumentam o passo de adaptação, levando a uma maior velocidade de convergência, enquanto valores baixos de erro reduzem o passo de adaptação, resultando em um menor desajuste do algoritmo [10].

Em geral, o algoritmo de Kwong é fortemente dependente do ruído aditivo, degradando o seu desempenho em ambientes com razão sinal-ruído (SNR) baixa.

\section{B. Algoritmo de Aboulnasr}

Este algoritmo é uma versão modificada do algoritmo de Kwong [10], baseando o ajuste do passo na medida de autocorrelação entre $e(n)$ e $e(n-1)$, ao invés de usar o erro quadrático instantâneo $e^{2}(n)$ [13]. Essa abordagem fornece uma razoável imunidade ao ruído aditivo de medição não-correlacionado. A atualização do passo de adaptação é baseada no quadrado da estimativa da autocorrelação do sinal de erro, obtida por

$$
p(n)=\beta p(n-1)+(1-\beta) e(n) e(n-1) .
$$

Essa operação representa uma filtragem passa-baixas, onde $\beta>0$ é um parâmetro de controle da filtragem. A expressão de atualização do valor do passo é então dada por

$$
\mu(n+1)=\alpha \mu(n)+\gamma p^{2}(n)
$$

onde $\alpha$ e $\gamma$ são parâmetros positivos de controle.

\section{Algoritmo Proposto}

A utilização da função de autocorrelação entre $e(n)$ e $e(n-1)$, apresentada em [13], pode (em algumas situações) não ser a melhor forma para efetuar o ajuste do passo de adaptação. Dependendo da correlação do sinal de entrada e do tipo de resposta ao impulso do sistema a ser identificado, a autocorrelação entre $e(n)$ e $e(n-2), e(n)$ e $e(n-3)$ e outros valores de lag pode ser tão ou mais significativa do que a autocorrelação do erro $\operatorname{lag}(1)$. Assim, no algoritmo de Aboulnasr [13], podem ocorrer situações em que o passo de adaptação seja reduzido prematuramente, deixando $o$ algoritmo desnecessariamente mais lento do que o esperado.

$\mathrm{O}$ algoritmo aqui proposto visa utilizar $N$ autocorrelações do erro. Com isso, o processo de convergência é acelerado, mantendo uma maior imunidade com vistas ao ruído de medição.

Então, consideramos agora $p(n)$, o valor médio da soma dos quadrados das funções de autocorrelação entre $e(n) \mathrm{e}$ $e(n-1), e(n-2), \ldots, e(n-N)$, estimado através da seguinte expressão:

$$
p(n)=\beta p(n-1)+(1-\beta) \sum_{i=1}^{N}[e(n) e(n-i)]^{2}
$$

onde $\beta>0$ é um parâmetro de controle de filtragem.

A equação de adaptação do passo variável é então dada por

$$
\mu(n+1)=\alpha \mu(n)+\gamma p(n)
$$

onde $\alpha$ e $\gamma$ são parâmetros de controle de ajuste do passo de adaptação. 


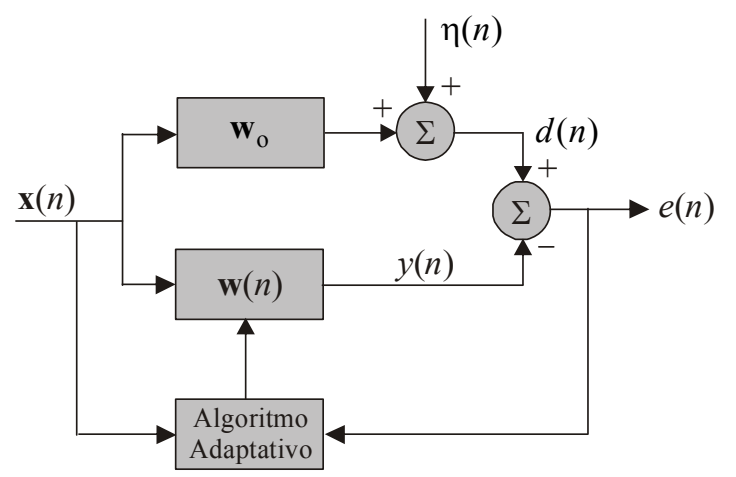

Fig. 1 - Diagrama em blocos de um esquema de identificação de sistema.

\section{Resultados de SimulaçÃo}

Nesta seção, são apresentadas simulações numéricas que comparam o desempenho do algoritmo proposto com os algoritmos de Kwong e de Aboulnasr. Para tal, um problema de identificação de sistemas é considerado, usando o mesmo cenário descrito em [13]. As figuras apresentadas mostram a evolução do passo de adaptação, a evolução de um coeficiente do vetor $\mathbf{w}(n)$ e o erro quadrático médio (EQM) em excesso dado por $E\left\{[e(n)-\eta(n)]^{2}\right\}$. Também, é observado o comportamento do algoritmo frente a uma mudança abrupta no sistema a ser identificado. A planta utilizada contém 4 coeficientes, dados pelo vetor $\mathbf{w}_{\mathbf{o}}=\left[\begin{array}{llll}5 & 0 & 1 & 8\end{array}\right]^{\mathrm{T}}$. Nas simulações, são considerados sinais de entrada branco e colorido, este último obtido através de um processo $\mathrm{AR}(1)$ dado por $x(n)=a x(n-1)+u(n)$, para $a=0,9, \sigma_{x}^{2}=5,26$ e $u(n)$ sendo um ruído branco gaussiano com variância $\sigma_{u}^{2}=1$. A dispersão dos autovalores da matriz de autocorrelação de entrada é $\chi=57,4$ e a variância do ruído aditivo é $\sigma_{\eta}^{2}=1$.

\section{A. Exemplo 1}

Neste exemplo, é utilizado um sinal de entrada branco com razão sinal-ruído (SNR) de $0 \mathrm{~dB}$. Os resultados numéricos são obtidos através de simulações Monte Carlo (MC), considerando 200 rodadas independentes. Os resultados dos algoritmos de Kwong, de Aboulnasr e o algoritmo proposto neste trabalho são comparados. Para todos os algoritmos, o passo máximo de adaptação é limitado a 0,1. As Figs. 2, 3 e 4 mostram os comportamentos do passo de adaptação, do coeficiente $w_{1}(n)$ e do EQM em excesso, respectivamente. Na Fig. 5, é ilustrado o comportamento do EQM em excesso considerando uma mudança abrupta nos parâmetros da planta. Essa mudança é obtida pela multiplicação dos coeficientes da planta por -1 na iteração 20000. A partir dos resultados apresentados nas figuras, verifica-se um melhor desempenho do algoritmo proposto. Em todas as simulações deste exemplo, os parâmetros dos três algoritmos são ajustados conforme os valores indicados na Tabela I, objetivando fixar o mesmo EQM final em excesso igual a $-33 \mathrm{~dB}$.
TABELA I

VALORES DOS PARÂMETROS DE CONTROLE

\begin{tabular}{c|l}
\hline \hline Algoritmos & Parâmetros \\
\hline \hline \multirow{2}{*}{ Kwong } & $\alpha=0,97$ \\
& $\gamma=7 \times 10^{-6}$ \\
\hline \multirow{3}{*}{ Aboulnasr } & $\alpha=0,97$ \\
& $\beta=0,99$ \\
& $\gamma=8 \times 10^{-4}$ \\
\hline Proposto & $\alpha=0,97$ \\
& $\beta=0,99$ \\
\hline \hline
\end{tabular}

\section{B. Exemplo 2}

Neste exemplo, é utilizado um sinal de entrada colorido com SNR igual a 7,2 dB. Novamente, uma comparação entre os algoritmos de Kwong, Aboulnasr e o aqui proposto é efetuada. Para todos os algoritmos, o passo máximo de adaptação é limitado a 0,01. As Figs 6,7 e 8 mostram a evolução do passo de adaptação, do coeficiente $w_{1}(n)$ e do EQM em excesso, respectivamente. A Fig. 9 ilustra a situação de uma mudança abrupta na planta (obtida de forma idêntica ao Exemplo 1), mostrando o comportamento do EQM em excesso para os algoritmos considerados. Os resultados numéricos obtidos ratificam o melhor desempenho do algoritmo proposto, quando comparado com as abordagens de Kwong e de Aboulnasr. Nas simulações deste exemplo, os parâmetros dos três algoritmos são ajustados de forma a se obter o mesmo EQM final em excesso igual a $-24 \mathrm{~dB}$. A Tabela II apresenta os valores usados para os parâmetros de controle dos algoritmos.

TABELA II

VALORES DOS PARÂMETROS DE CONTROLE

\begin{tabular}{c|l}
\hline \hline Algoritmos & Parâmetros \\
\hline \hline \multirow{2}{*}{ Kwong } & $\alpha=0,97$ \\
& $\gamma=1,1 \times 10^{-5}$ \\
\hline \multirow{3}{*}{ Aboulnasr } & $\alpha=0,97$ \\
& $\beta=0,99$ \\
& $\gamma=1,6 \times 10^{-3}$ \\
\hline \multirow{2}{*}{ Proposto } & $\alpha=0,97$ \\
& $\beta=0,99$ \\
& $\gamma=3 \times 10^{-6}$ \\
\hline \hline
\end{tabular}

\section{CONCLUSÕES}

Neste trabalho, um novo algoritmo LMS de passo variável para ambientes com baixa razão sinal-ruído é proposto. $\mathrm{O}$ novo algoritmo considera a função de autocorrelação do sinal de erro para $N$ lags. O desempenho do algoritmo proposto, em aplicações com alto nível de ruído, é superior ao de outros algoritmos LMS de passo variável similares. Resultados de simulações numéricas confirmam a eficácia da estratégia proposta tanto para sinal de entrada branco quanto colorido. 


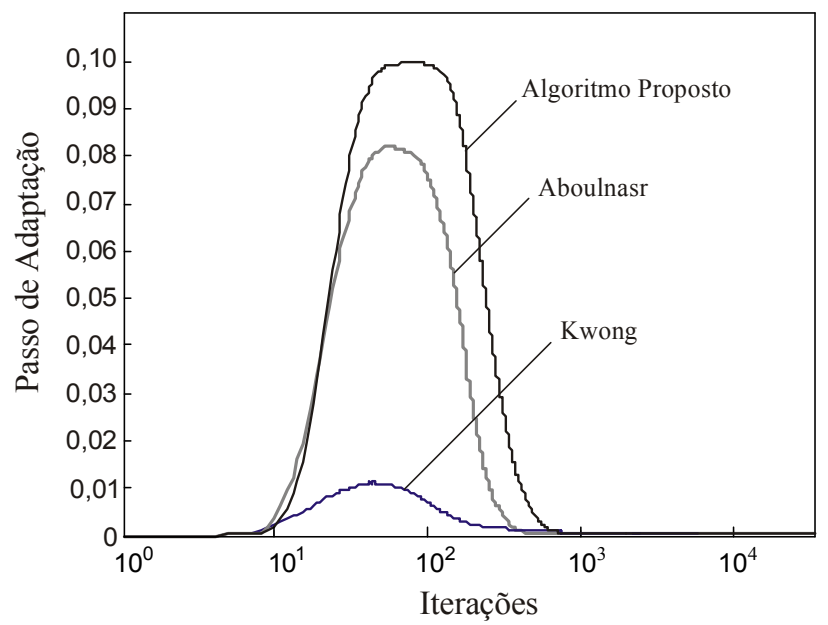

Fig. 2. Evolução do passo de adaptação para sinal de entrada branco.

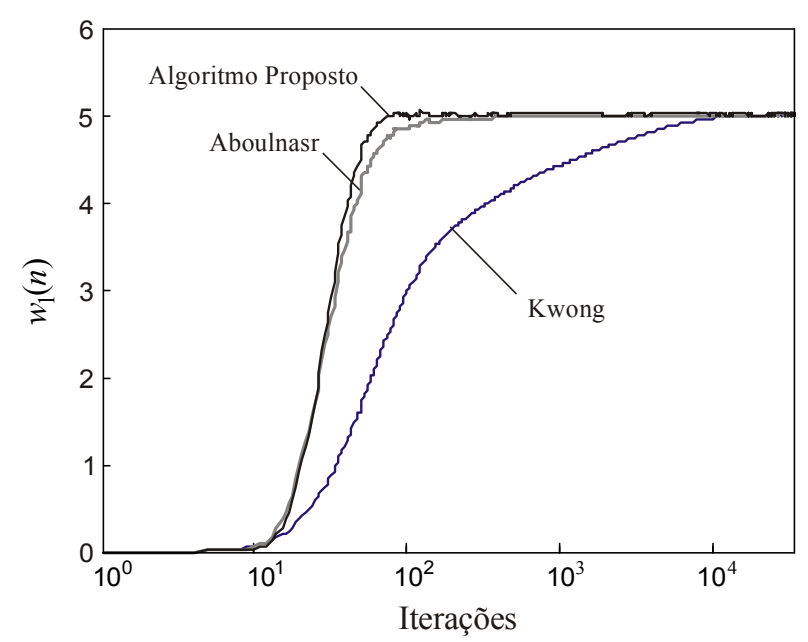

Fig. 3. Evolução de $w_{1}(n)$ para sinal de entrada branco.

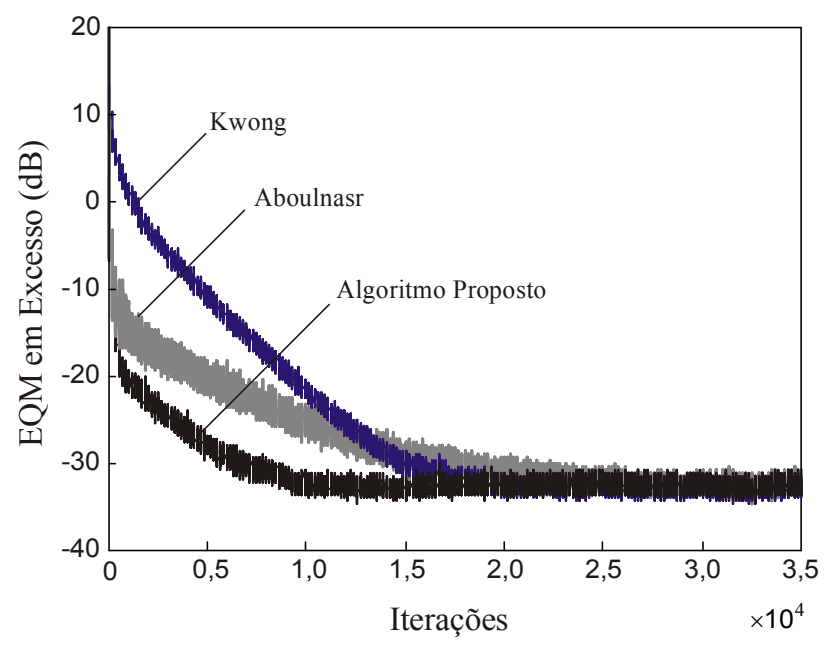

Fig. 4. Comportamento do EQM em excesso para sinal de entrada branco.

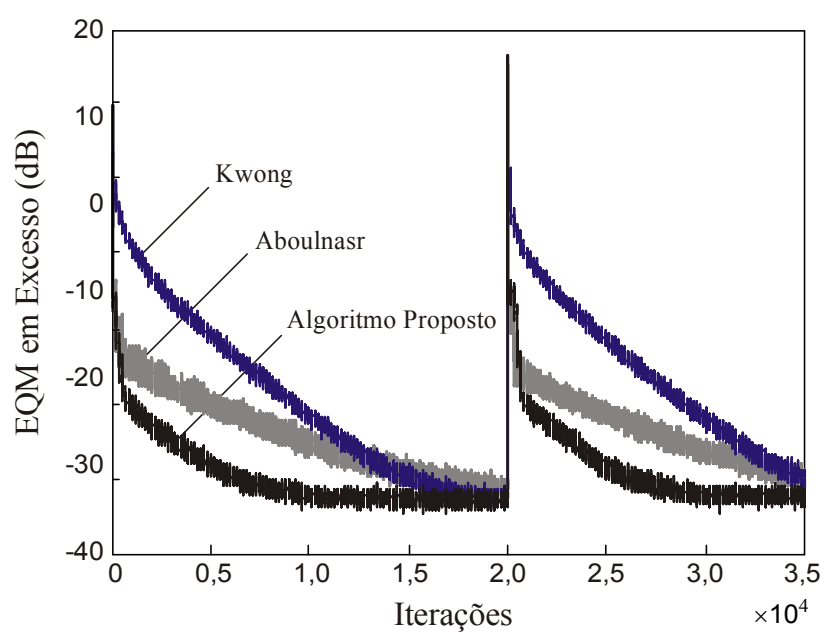

Fig. 5. Comportamento do EQM em excesso para sinal de entrada branco com uma mudança abrupta na planta na iteração 20000.

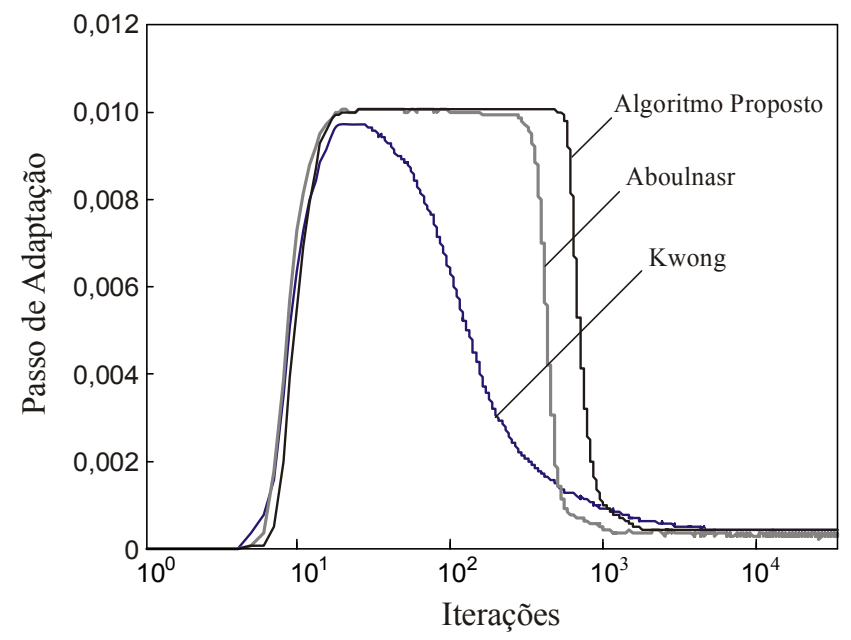

Fig. 6. Evolução do passo de adaptação para sinal de entrada colorido.

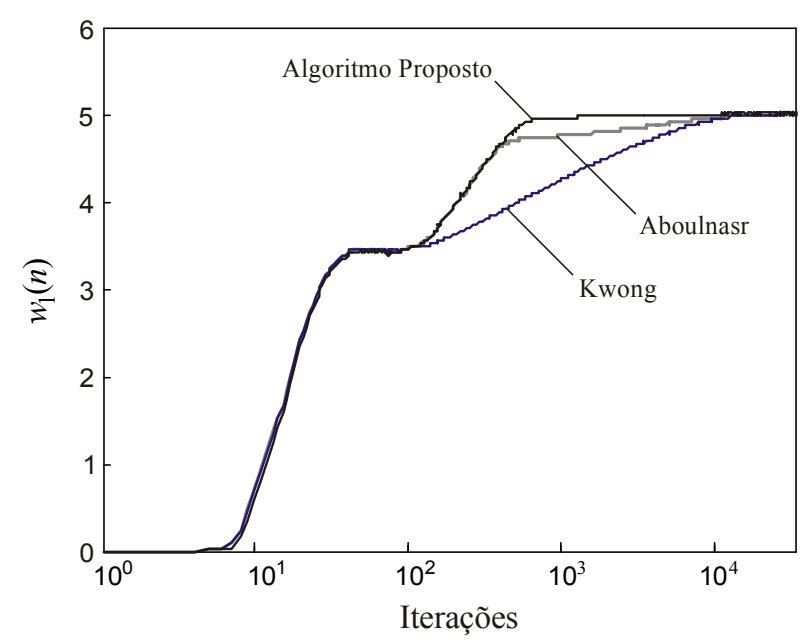

Fig. 7. Evolução de $w_{1}(n)$ para sinal de entrada colorido. 


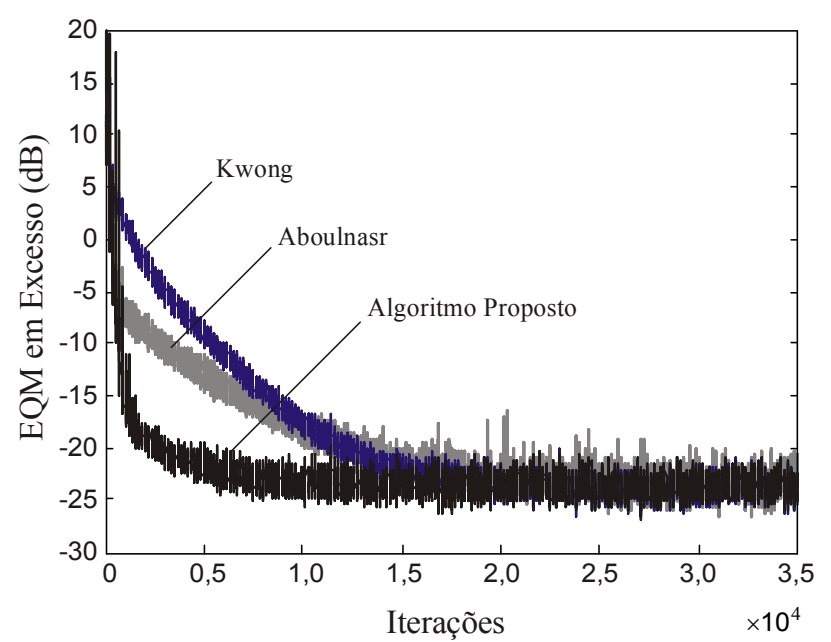

Fig. 8. Comportamento do EQM em excesso para sinal de entrada colorido.

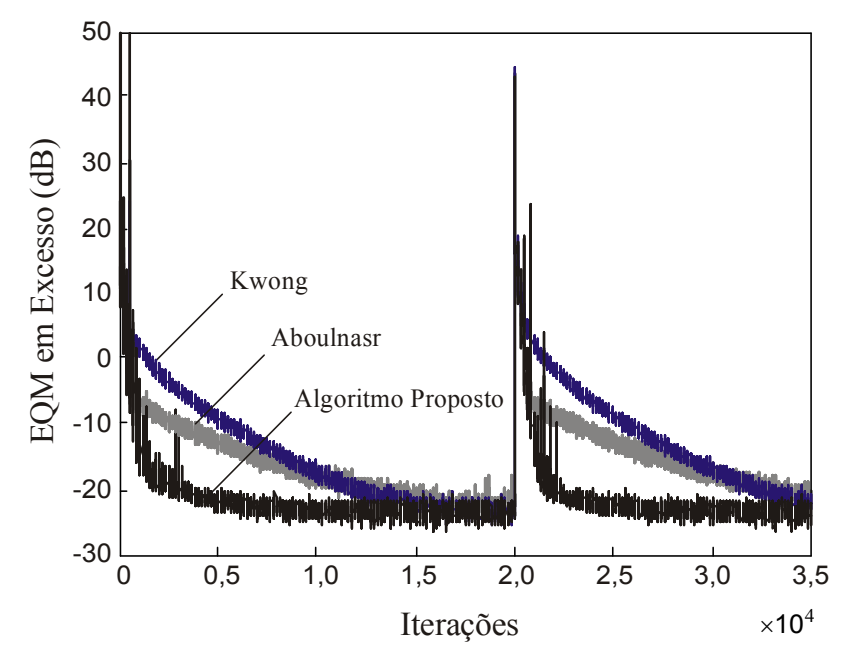

Fig. 9. Comportamento do EQM em excesso para sinal de entrada colorido com uma mudança abrupta na planta na iteração 20000.

\section{REFERENNCIAS}

[1] B. Widrow and M. Hoff, "Adaptive switching circuits," in Proc. IRE Western Electronic Show and Convention, New York, USA, Part 4, Aug. 1960, pp. 96-104.

[2] S. Haykin, Adaptive Filter Theory, $4^{\text {th }}$ ed., Upper Saddle River, NJ: Prentice Hall, 2002.

[3] J. C. Richards, M. A. Webster, and J. C. Principe, "A gradient-based variable step-size LMS algorithm," in Proc. IEEE Southeastcon, Williamsburg, USA, vol. 2, Apr. 1991, pp. 1083-1087.

[4] V. J. Mathews and Z. Xie, "A stochastic gradient adaptive filter with gradient adaptive step size," IEEE Trans. Signal Process., vol.41, no. 6, pp. 2075-2087, June 1993.

[5] A. I. Sulyman and A. Zerguine, "Convergence and steady state analysis of a variable step-size normalized LMS algorithm," Proc. IEEE Int. Symp. Signal Processing and Its Applications (ISSPA), Paris, France, vol. 2, July 2003, pp. 591-594.

[6] B. Farhang-Boroujeny, "Variable step size LMS algorithm - New developments and experiments," IEE Proceedings - Vision, Image, Signal Process., vol. 141, no. 5, pp. 311-317, Oct. 1994.

[7] T. J. Shan and T. Kailath, "Adaptive algorithms with an automatic gain control feature," IEEE Trans. Circuits Syst., vol. CAS-35, no. 1, pp. 122-127, Jan. 1988
[8] J. Okello, Y. Itoh, Y. Fukui, I. Nakanishi, and M. Kobayashi, "A new modified variable step size for the LMS algorithm," in Proc. IEEE Int. Symp. Circuits and Systems (ISCAS), Monterey, USA, vol. 5, Jun. 1998, pp. 170-173.

[9] W. P. Ang and B. Farhang-Boroujeny, "A new class of gradient adaptive step-size LMS algorithms," IEEE Trans. Signal Process., vol. 49, no. 4, pp. 805-810, Apr. 2001.

[10] R. H. Kwong and E. W. Johnston, "A variable step size LMS algorithm," IEEE Trans. Signal Process., vol. 40, no. 7, pp. 1633-1642, July 1992.

[11] I. Nakanishi and Y. Fukui, "A new adaptive convergence factor algorithm with the constant damping parameter," IEICE Trans. Fundamentals, vol. E78-A, no. 6, pp. 649-655, Jun. 1995.

[12] M. H. Costa and J. C. M. Bermudez, "A robust variable step size algorithm for LMS adaptive filters," in Proc. IEEE Int. Conf. Acoust., Speech, Signal Process., Toulouse, France, vol. 3, May 2006, pp. 93-96.

[13] T. Aboulnasr and K. Mayyas, "A robust variable step-size LMS-type algorithm: analysis and simulations," IEEE Trans. Signal Process., vol. 45, no. 3, pp. 631-639, Mar. 1997.

[14] D. W. Kim, J. H. Hoi, Y. S. Choi, C. H. Jeon, and H. Y. Ko, "A VS-LMS algorithm using normalized absolute estimation error," in Proc. IEEE Digital Signal Processing Applications (TENCON), Perth, Australia, vol. 2, Nov. 1996, pp. 692-697.

[15] Z. Ramadan and A. Poularikas, "A robust variable step-size LMS algorithm using error-data normalization," in Proc. IEEE Southeastcon, Huntsville, USA, Apr. 2005, pp. 219-224.

[16] B. Rohani and K. S. Chung, "A modified LMS algorithm with improved convergence," in Proc. IEEE Singapore Int. Conf. Communication Systems, Singapore, Nov. 1994, pp. 845-849.

[17] D. L. Duttweiler, "Proportionate normalized LMS adaptation in echo cancellers," IEEE Trans. Speech Audio Process., vol. 8, no. 5, pp. 508-518, Sept. 2000.

[18] J. Benesty and S. L. Gay, "An improved PNLMS algorithm," in Proc. IEEE Int. Conf. Acoust., Speech, Signal Process., Orlando, USA, May 2002, pp. 1881-1884.

[19] Y. Wei and S. B. Gelfand, "Noise-constrained least mean squares algorithm," IEEE Trans. Signal Process., vol. 49, no. 9, pp. 1961-1970, Sept. 2001. 